\title{
Some Investigations of the Remanent Magnetism and Domain Structures of Iron Meteorites
}

\author{
Robert L. DuBoIs \\ Department of Geology, University of Arizona, Tucson, Arizona, U.S.A.
}

\begin{abstract}
The direction and intensity of remanent magnetism has been measured on a series of iron meteorite specimens and their magnetic domain patterns have been studied on polished surfaces. Specimens were carefully collected in the field and their geographic orientation noted. From these specimens, the magnetic effects of the geomagnetic field could be estimated and only those suecimens which lacked such effects were used for further study. Specimens obtained from museums or similar sources are not considered satisfactory for these magnetic studies.

The intensity of remanent magnetism for octahedries from Arizona Crater averages $11.6 \times 10^{-3} \mathrm{emu} / \mathrm{cm}^{3}$, whereas that for octahedrites from Odessa Crater averages $6.6 \times 10^{-3}$ $\mathrm{emu} / \mathrm{cm}^{3}$. Domain patterns for schreibersite have a chain-like cross-section and are rod shaped in longitudinal section. Kamacite has a rectangular pattern when carefully prepared or under other conditions, a maze pattern.
\end{abstract}

\section{Introuction}

This paper reports the results of a series of experiments designed to contribute some knowledge on the remanent magnetism of iron meteorites. Some of the research gave negative results in that the measured properties were subsequently found to have been caused by conditions during collection or during laboratory preparation of samples for measurement. These results suggest some limitations as to sources of materials and also as to procedures used in sample preparation. Finally, significant measurements were made from which some tentative conclusion can be drawn.

The magnetic properties of iron metorites have been little studied by the scintific community. Important contributions have been made by Lovering (1959) and Lovering and Parry (1962) on iron meteorites and by Stacy and Lovering (1959) and Weaving (1962) on chondritic meteorites.

Iron meteorites are essentially iron-nickel alloys containing small amounts of other minerals. They are classified according to structure into hexahedrites, octahedrites and nickel-rich ataxites and the vraious groups are closely related to nickel content, (Mason, 1962). The hexahedrites contain 4-6 percent nickel and are largely made up of the mineral kamacite (body-centered cubic lattice, alpha phase alloy). Octahedrites are composed of oriented kamacite and taenite (face-contered cubic lattice, gamma phase alloy) bands in octahedral arrangement. Their nickel content ranges from 6-14 percent. Ataxites have nickel contents in excess in 12 percent and are composed mainly of either plessite (intergrowth of kamacite 
and taenite) or taenite. Besides kamacite and taenite another mineral present in iron meteorites that is important to magnetic studies studies is schreibersite, an iron-nickel phosphide that occurs as rounded grains or as shells around nodules of troilite (iron sulfide).

\section{Sample Collection, Preparation, and Measurement}

Specimens for this investigation were obtained from the Geology Department Museum at the University of Arizona and from the Nininger Meteorite collection at Arizona State University. In addition, specimens were collected in the field from Arizona Crater (Canyon Diablo Crater) and Odessa Crater (Odessa, Texas). The field location of specimens was made through the aid of a war surplus mine detector. After a specimen was located, the "soil" overburden was carefully removed, care being exercised to assure that the specimen was not struck by any metal digging tools. It had been shown early in the investigations that striking the meteorite by a blow from a metal object sometimes incrased the intensity of magnetism by several orders of magnitude. In most cases final digging and removal was accomplished by the hands alone. When the specimen was found to be an adequate size for orientation, care was exercised during the final stages of digging to not disturb it until an orientation marker was attached to a suitable location on the specimen. The marker consisted of a piece of adhesive tape upon which were placed strike and dip lines and their value of attitude. The lines were located on the tape with a small hand level and the strike was determined by transfer of a magnetic azimuth and dip by a clinometer. Only the specimens that, because of their location or nature of burial were considerd likely to have not moved since original emplacement, were oriented. Many specimens from near the crater rims have obviously moved during creep of their enclosing material, and are not suitable for orientation. Those near the present surface or near man-made excavations also have probably been disturbed. The most satisfactory setting seems to be large flat plains somewhat removed from the main crater and for specimens to be buried some 6 to 12 inches in the ground. Those specimens which were encased in caliche (natural secondary deposit of calcium carbonate) or had a thick oxide encasement which had discolored the nearby soil were considered especially suitable.

After removal from the ground, the specimens were carefully packed to protect them from shock and other accidents. Most museum specimens are unsatisfactory for these investigations because they either have been "tested" with small magnets, or as in many cases actually been collected using large field magnets. Their history between the time of collection and measuring is not known and therefore they are not suitable for these studies.

Magnetic measurements were made using an astatic magnetometer by an on-center method. Various procedures were used to prepare the specimens for measurements. Some of them were measured after only a cleaning with water whereas others had their thin oxide coating removed with $\mathrm{HCI}$ acid. Slabs approximately one-quarter of an inch thick were cut across some specimens using a hack saw and others using an abrasive impregnated rubberblade cooled with water. Some slabs were measured as single units while others were cut into small rectangular pieces. 
Thermal cleaning was accomplished in a bifilar wound electric furnace and specimens were enclosed in a vacuum bomb. A Helmholtz coil system was used to null the earth's field during the experiment and the amplitude of the residual field was recorded constantly. During the yeating and cooling cycles the residual field was less then 100 gammas.

For studies of domain patterns, the specimens were roughground by hand and then polished using a vibrating polisher. Electrolytic polishing was tried but with poor results. The domain patterns were obtained by placing a few drops of a ferromagnetic colloid (Elmore, 1938) on the specimen and covering the area with a thin cover glass.

\section{Experimental Results and Discussion of Studies of Remanent Madnetism}

The first sample selected for study was a slab one-quarter inch thick cut across a large specimen of the Weaver meteorite (ataxite). The slab was cut into 60 small rectangular pieces one-half inch square and one-quarter inch thick and the direction of the remanent magnetism was measured for each piece. The reference direction was chosen with the long axes of the slab as north-south and the plane of the slab as the horizontal. The results are plotted in Fignre 1 as a sterogram with solid circles representing points on the lower hemis-

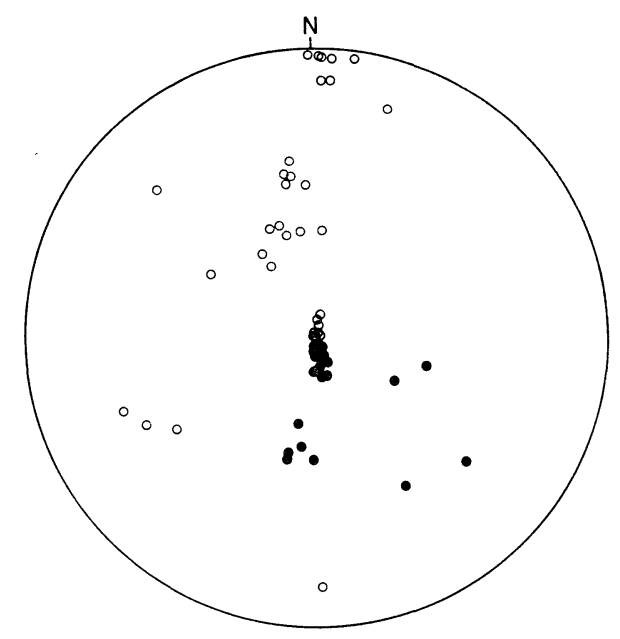

Fig. 1 Direction of remanent magnetism of rectangular pieces prepared from the Weaver meteorite.

phere and open circle points on the upper hemisphere. Two general directions of remanent magnetism are observed from the data; one with the north-seeking pole pointing southward and with a positve inclination (angles measured below the horizon), two with the northseeking pole to the north and with a negative inclination (angles measured above the horizon). There is of course some scatter but generally the results have considerable consistency. The two directions are difficult to interpretate when, from theoretical considerations, a single direction of polarization would be expected. The intensity of the remanent magnetism was measured and the results are plotted in Figure 2. The average value is $2.1 \mathrm{emu} / \mathrm{cm}^{3}$ and whereas the intensity is high it does not vary greatly in magnitude. 


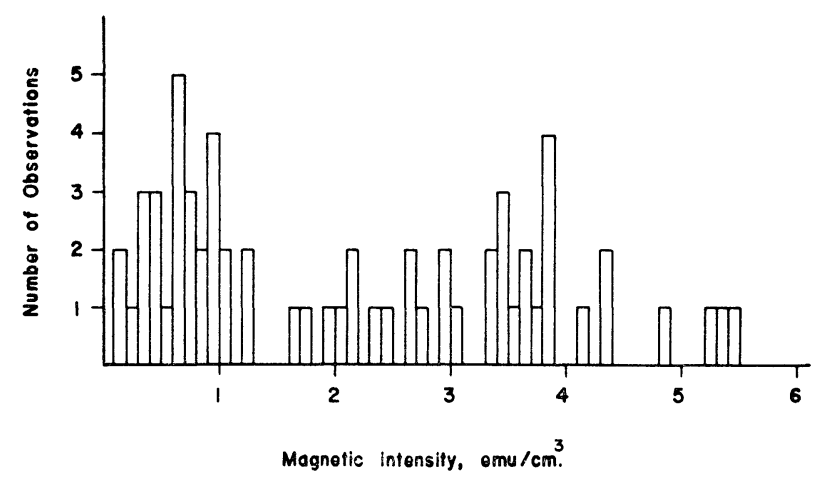

F!g. 2 Intensity of remanent magnetism of rectangular pieces prepared from the Weaver meteorite.

To determine if the normal-reverse magnetic polarization and the high value of intensity are characteristic of iron meteorites a second slab was similarly prepared and measured but from an octahedrite. The results of measurements of direction are presented in Figure 3. Again, exclusive of some scatter, there is some consistency to the results but not as much as in the results from the Weaver meteorite. The intensity of the remanent magnetism ranged from 1.4 to $186 \times 01^{-3} \mathrm{emu} / \mathrm{cc}$ and the average is $54 \times 10^{-3} \mathrm{emu} / \mathrm{cm}^{3}$.

The acquisition of results from the first two specimens measured and their general consistency seemed to be encouraging. To determine if the magnetism was acquired in the earth's field, slabs were prepared from 2 oriented octahedrites from Odessa. Each slab was further cut into three or four pieces to determine the internal consistency of the results. In Figure 4, the results of the measurements made on the three pieces from a single slab as referenced to the plain of the slab are a series along a great circle and about $180^{\circ}$ apart.

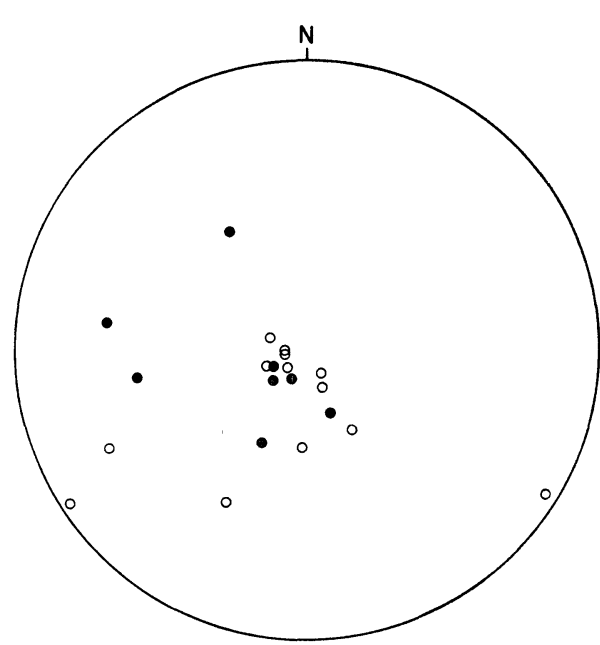

Fig. 3 Direction of remanent magnetism of rectangular pieces prepared from an octahedrite.

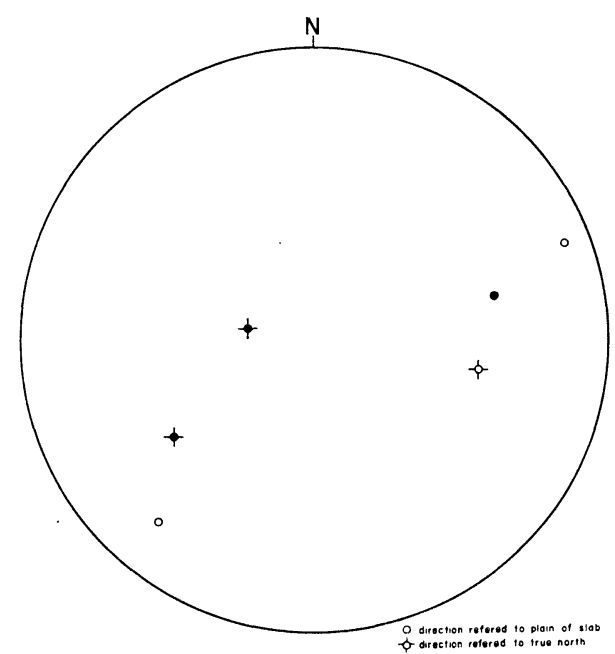

Fig. 4 Direction of remanent mganetism of three pieces prepared from a slab taken from an oriented Odessa meteorite. 
Again internal consistency and characteristics of direction similar to those of the Weaver meteorite are observed. The results from the measurements made on the four pieces of the second meteorite are given in Figure 5. The directions of magnetism as referenced to true north are also given in Figures 4 and 5 and these directions do not group near the present field of the earth and therefore it may be suggested that magnetization of these iron meteorites was not acquired in the earth's magnetic field at the sample site.

The results from the oriented slabs seemed encouraging and therefore 11 more slabs were prepared each from an oriented specimen. The specimens are all octahedrites from

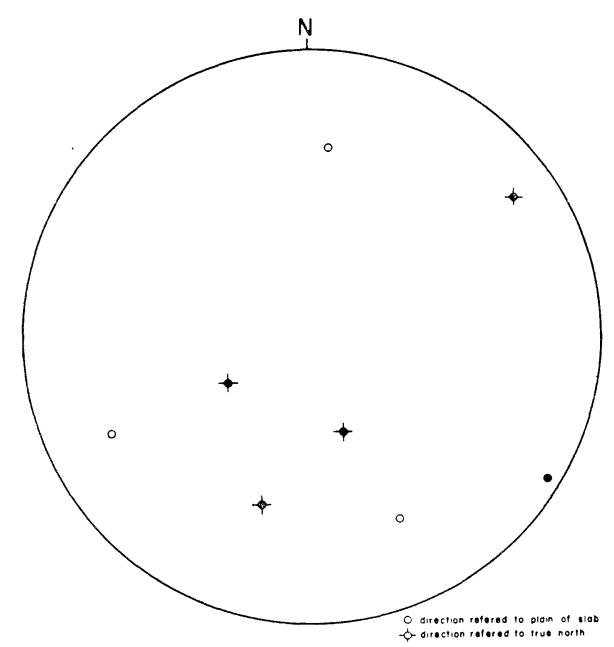

Fig. 5 Direction of remanent magnetism of pieces prepared from a slab taken from an oriented Odessa meteorite.

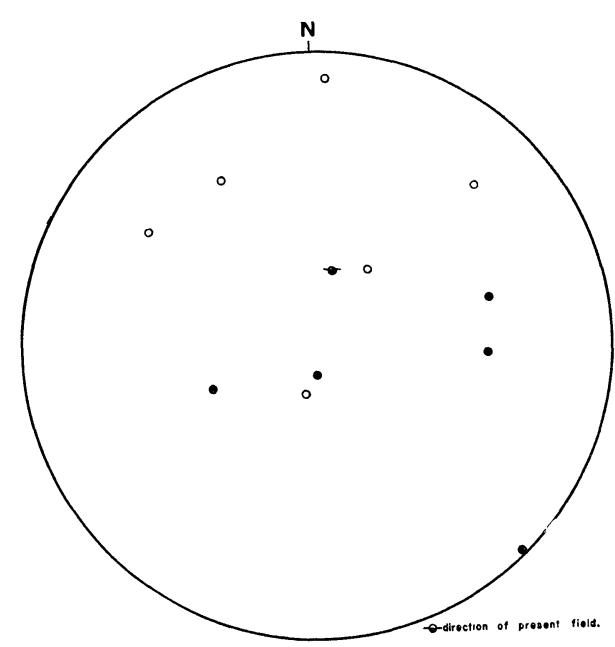

Fig. 6 Direction of remanent magnetism as refered to true north for slabs prepared from octahedrites from Odessa and Arizona craters.

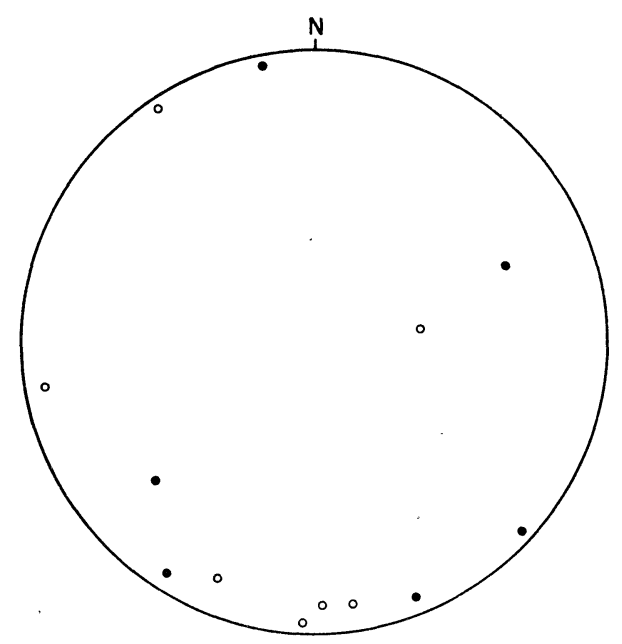

Fig. 7 Direction of remanet magnetism as refered to the plain of the slabs which have been prepared from octahedrites from Odessa and Arizona Craters. 
Odessa and Arizona Crater. The measured directions of remanent magnetism as referenced to true north are plotted in Figure 6 and as referenced to the plain of the slab in Figure 7. Figure 6 shows a lack of coincidence between the direction of remanent magnetism and the direction of the present field. From Figure 7, it can be seen that as most of the measured directions of remanence have small angles of inclination when referenced to the plain of the slab that the measured magnetization is in the plain of the slab. These results suggest that as the measured magnetism of the slabs having different original orientation in the field is not a random feature as should be expected from specimens magnetized under extraterrestrial conditions, it has been acquired during the preparation of the slabs.

Attention was then turned to individual oriented specimens and measurements made on them in their original form. Those used were selected for their approximation to a sphere. The results are given in Figure 8 where the directions of magnetization can be referenced either

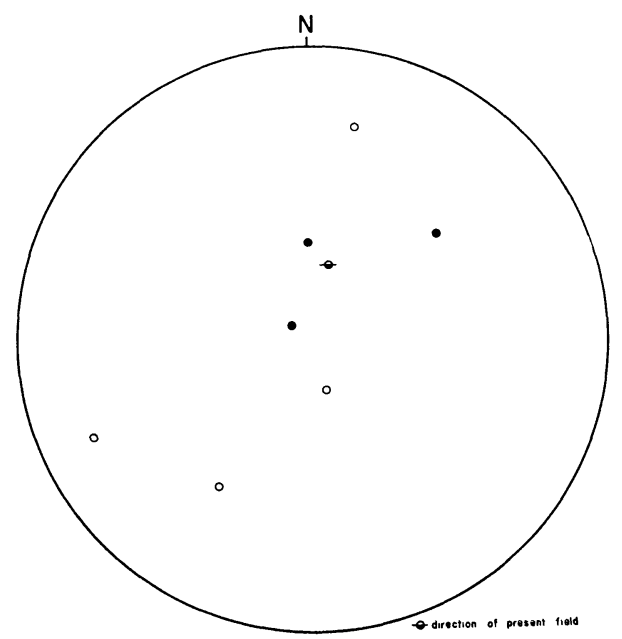

Fig. 8 Direction of remanent magnetism of oriented octahedrite specimens from Odessa and Arizona Craters.

to the direction and plain of orientation of the specimen or to true north. The results when referenced to true north suggest that three specimens have directions of remanent magnetism similar to that of the existing earth's field, whereas four are quite dissimilar. The magnetism of the specimens with directions near that of the earth's field could be related to the earth's field or else the similarity of their directions with that of the earth's field could be a coincidence. The magnetism of the others is tentatively concluded to be extra-terrestrial in origin.

A group of small unoriented specimens were measured as collected for intensity of remanent magnetization. Ten such specimens from Arizona Crater gave valuse of intensity ranging from 1.5 to $28.4 \times 10^{-3} \mathrm{emu} / \mathrm{cc}$ and averaged $11.6 \times 01^{-3} \mathrm{emu} / \mathrm{cm}^{3}$. The distribution of values is given in Figure 9. Similarly 14 specimens from Odessa crater ranged in intensity from 2.6 to $19.4 \times 10^{-3} \mathrm{emu} / \mathrm{cm}^{3}$ (Figure 10) and averaged $6.6 \times 10^{-3} \mathrm{emu} / \mathrm{cc}$. The consistency of the values of intensity for the various specimens from either crater (Figures 9 and 10) 


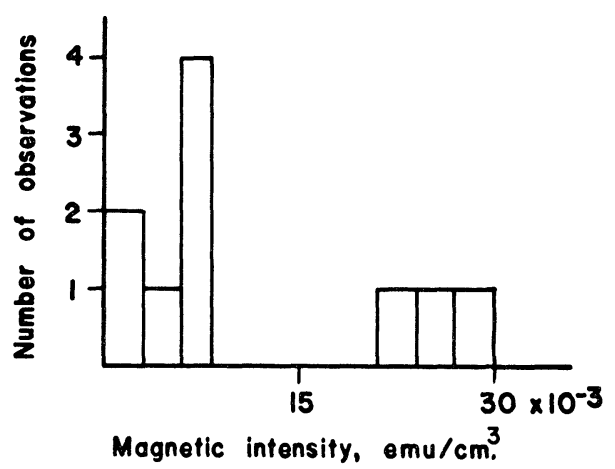

Fig. 9 Intensity of remanent magnetism of octahedrites from Arizona Crater.

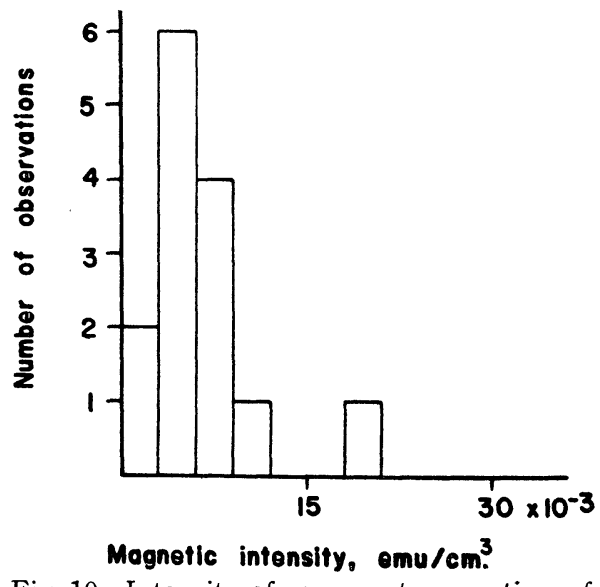

Fig. 10 Intensity of remanent magnetism of octahedrites from Odessa Crater.

seems to suggest that the magnetization of the specimens has occurred under very uniform conditions such as those expected under extraterrestrial or terrestrial fields. The similarity of the average values for the two groups may be important but these results must first be compared to ones from other craters. Lovering (1959) has published some values of intensity of remanent magnetism for 3 different specimens of octahedrites which range in intensity from 16.1 to $60.3 \times 10^{-5} \mathrm{emu} / \mathrm{cm}^{3}$ and for one ataxite specimen he gives a value of intensity of $439 \times 10^{-5}$. The values for the octahedrites are lower than those obtained in this study by a factor of 10 , and their lower values might lend support to the argument that the similarity of values of intensity obtained from specimens from Arizona and Odessa craters is significant and that they may be the same fall. More measurements are needed from other areas.

The average value of intensity for the specimens from Odessa is smaller by a factor of 2.4 than that of the measured slabs prepared from specimens from the same crater. The corresponing value is lower by 2.8 times that obtained for the average intensity of the slabs of specimens from Arizona Crater. The ratios 2.4 and 2.8 give some indication of the magnitude of secondary magnetization imparted by slabing. The results of intensity measurements made on the Weaver meteorite probably cannot be directly compared because of a difference in the composition of the meteorites. The results of intensity measurements made on the oriented specimens used to obtain the direction data of Figure 8 are of the same magnitude as that used in Figures 9 and 10.

To study the distribution of secondary magnetism of the meteorites and to determine the magnitude of the magnetism associated with a thin oxide coating present on meteorite specimens, chemical leaching experiments were made on a prepared slab, on an oriented specimen, and on 3 small indivdual unoriented specimens. The slab initially underwent a decrease of intensity by a factor of 5 with a $20 \%$ reduction in weight and a second $20 \%$ reduction in weight caused only a decrease of $25 \%$ in intensity. These results suggest that the secondary magnetization present in slabs is mainly present in the near surface material. The oxide coating of three unoriented specimens was leached away and the specimens lost 
about $30 \%$ in weight. They underwent erratic changes in magnetic intensity and no uniform magnetic contribution by the oxide coating on the magnetization could be determined. The direction of remanent magnetism of the oriented specimen was little changed after a chemical leaching involving a weight loss of 20 percent and, therefore, the magnetism of the oxide coating contributed only minor amounts to the total magnetism.

Thermal magnetic cleaning was tried on three of the slab specimeds to remove some of the components of secondary magnetization. Heating to $125^{\circ} \mathrm{C}$ of one specimen caused only a $10 \%$ decrease in intensity and a shift of only $7^{\circ}$ indirection, whereas the other two specimens lost $50 \%$ of their original intensity and changed directions by $57^{\circ}$ and $53^{\circ}$. Complete thermal magnetic analyses are now in progress.

\section{Magnetic Domain Studies}

It is desirable to further test the idea of an extra-terrestrial origin for the magnetism of some of the meteorites. From consideration of the curie temperatures of the various mineral phases present and of the possible relative times of magnetization, magnetic domain pattern studies offer one approach. The curie temperature for a sample of schreibersite has been measured at $310^{\circ} \mathrm{C}$ by Lovering and Parry (1962). Kamacite is a reversible alloy which upon heating transforms from magnetic alpha phase to non-magnetic gamma phase at temperatures which are dependent upon composition. The curie point of alpha phase is for some compositions above the transformtion temperature. In either case magnetization of kamacite, booled below curie or transformtion temperatures, occurs at relatively high temperatures, upwards of $700^{\circ} \mathrm{C}$. Now if meteorites are derived from a primary mass that had cooled to a tempesature of about $700^{\circ} \mathrm{K}$ and then disrupted as suggested by Lovering (1957), the magnerization of kamacite should have occurred before disruption and that of schreibersite afterwards. In these respects the direction of magnetization of the two mineral types would be different and the occurrence of different directions for their magnetization would lend some support to the conclusion that the magnetization was at least in part extraterrestrial in origin.

To undertake preliminary studies of domain structure, magnetic domain patterns were obtained on various specimens from both the Odessa and Arzoina Craters. The patterns obseved on different grains of the same mineral are found to be the same for all occurrences of that mineral examined from either crater. The individual patterns for the two different minerals studied are, however, quite different and magnetic domain analysis offers a method for their identification in iron meteorites.

The mineral schreibersite has a characteristic chain-like domain pattern in cross section and a rod-like form in longitudinal section (Figures 11 and 12). Distortions of the domain patterns are observed near inclusions or grain boundries. Kamacite has an entirely different pattern (Figure 13) from schreibersite and one which resembles the maze pattern obtained by Bozorth (1951) on iron crystals. For some patterns on kamacite (Figure 14) the application of a strong magnetic field caused movement of the domain walls and, heretfore, this pattern is not considered to be a maze pattern. Figure 14 relates the shape of the domain 


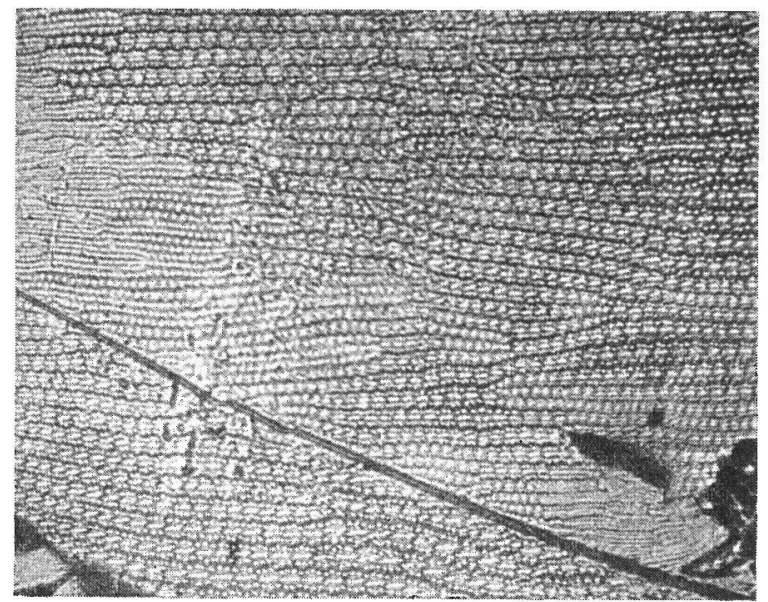

Fig. 11 Magnetic domain pattern obtained on schreibersite.

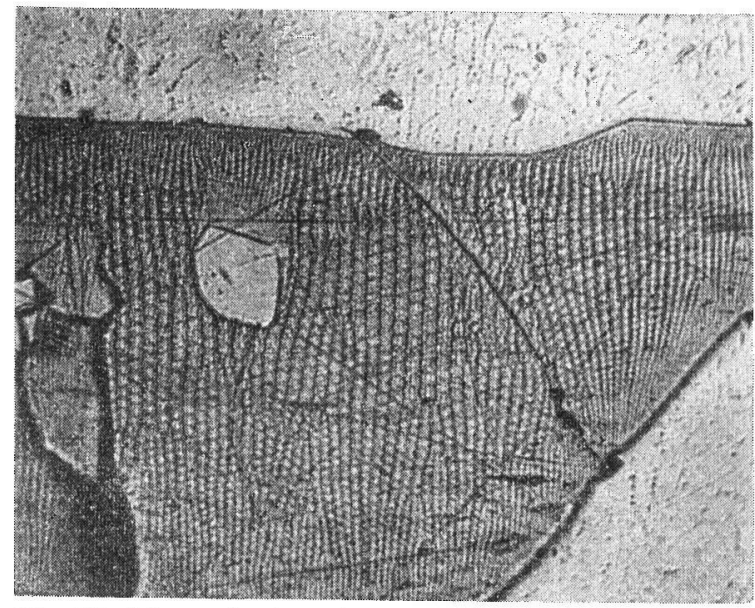

Fig. 12 Magnetic domain pattern obtained on schreibersite.

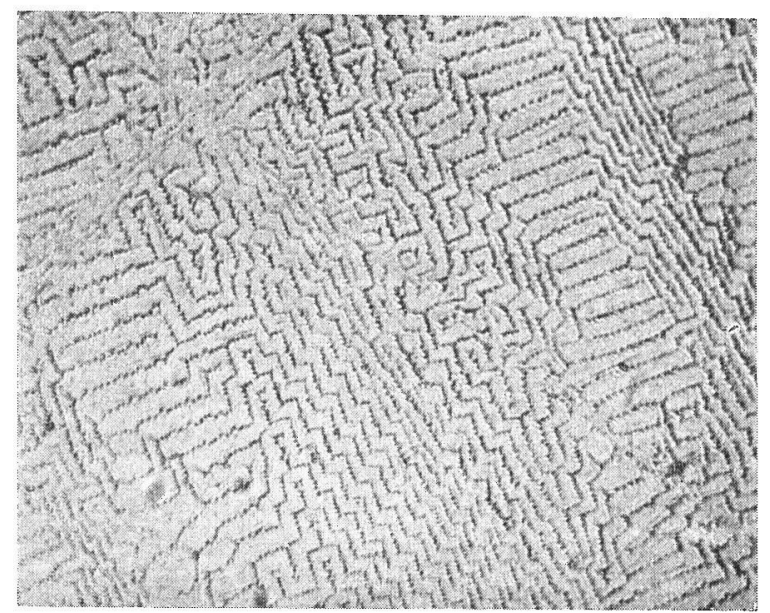

Fig. 13 Magnetic domain pattern obtained on kamacite. 


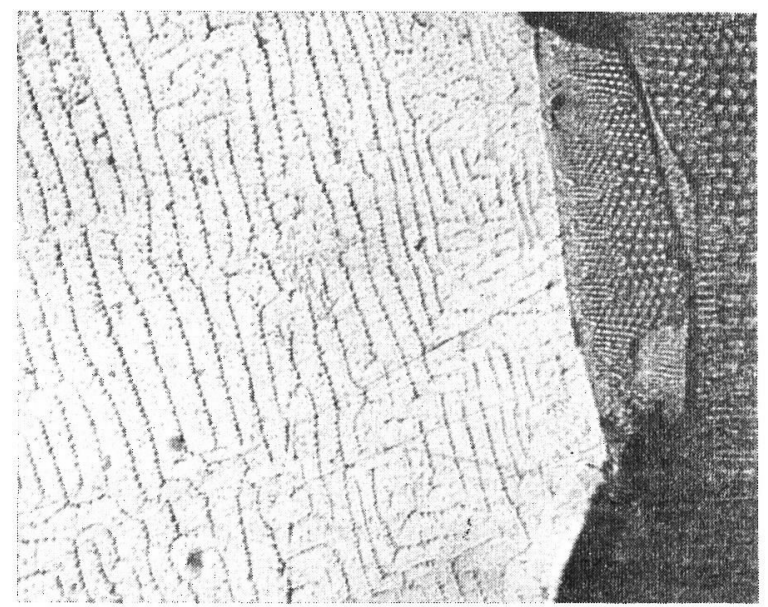

Fig. 14 Magnetic domain pattern obtained on kamacite.

pattern for kamacite to that of schreibersite and also to the measured direction of the remanent magnetism. The long dimension of the pattern for schreibersite is at an angle to the direction of the pattern for kamacite and is nearly pararllel to the measured direction for remanence. A lack of coincidence of the two directions supports the suggestion that their magnetization is at least in part extra-terrestrial in origin. More similar studies are needed on meteorites from these and other areas.

\section{Acknowledgments}

I wish to thank Professors J.W. Anthony and S.R.. Titley of the Gsology Department, University of Arizona, for many discussions and for reading the manuscript. Acknowledgments are also given numerous graduate students for their help in the collection of some of the specimens and in making measurements. My appreciation is extended to Mr. T. Rodman and to Odessa Meteoritical Society of Odessa, Texas for help in obtaining specimens of Odessa meteorites and Mr. Thompson of the Arizona Meteor Crater for permission to collect. H.H. Nininger and Dr. C.B. Moore accompanied the author on a collecting trip to Arizona Crater and aided greatly in the collecting of specimens.

The research was partly supported by the National Science Foandation under grant GP-1455.

\section{References}

Elmore, W.C., Ferromagnetic colloid for studying magnetic structure, Phys. Rev. 54, 309-310, 1938.

Lovering, J.F., Pressures and temperatures within a typical parent meteorite body, Genchim. et Cosmochim., 12, 253-261, 1957.

Lovering, J.F., The magnetic field in a primary meteorite body, Am. Soc. Sci., 257, 271-275 1959.

Lovering, J.F. and Parry, L.G., Thermomagnetic analysis of co-existing nickel-iron metal phases in iron meteorites and the thermal histories of the meteorites, Geochim. et Cosmochim., Acta, 26, 362-382, 1962. Stacey, F.D., and Lovering, J.F., Natural magnetic momentf of two Chondritic Meteorites, Nature, 183, 529-530, 1959.

Weaving, B., Mangetic anistropy in Chondritic Meteorites, Geochim. et Cosmochim., 26, 45โ-455, 1962. Mason, B., Meteorites, J. Wiley, N.Y., 1962. 